\title{
Cómo y dónde se encuentra la profesión médica. Visión de un médico especialista en formación.
}

\author{
Pablo Ryan Murúa \\ Departamento de Medicina Interna. Hospital Universitario Gregorio Marañón. Madrid.
}

Probablemente no hay otro tema tan interesante y polémico como el "cambio social". Este es un tema de interés en muchos campos del saber, particularmente en salud. A pesar de ser este término muy utilizado, no hay una comprensión unánime de este concepto.

Hoy, la profesión médica se enfrenta a la explosión tecnológica, al cambio en las fuerzas del mercado, a problemas en la prestación de servicios sanitarios, al bioterrorismo y a la globalización. Como resultado de todo ello, los médicos encuentran que cada vez es más difícil cumplir sus responsabilidades con los pacientes y con la sociedad. En estas circunstancias, lo importante es reafirmar los principios y valores fundamentales y universales de la profesión médica, que siguen siendo los ideales a perseguir por todos los médicos.

Este cambio social se debe en gran medida a una serie de fenómenos sociales que se pueden clasificar como demográficos, epidemiológicos, tecnológicos, económicos, laborales, políticos y éticos entre otros.

Cambios demográficos: El aumento de la longevidad de la población, la baja tasa de natalidad y el incremento de la inmigración procedente de otros países. El aumento de la longevidad de la población ha supuesto un incremento de las necesidades sanitarias, tanto por el diagnóstico de nuevas enfermedades relacionadas con el hecho de vivir más años como por un aumento de la frecuentación de los servicios de salud. Así mismo, los conceptos de "cuidar" y de "calidad de vida" aparecen asociados al desarrollo de servicios sociosanitarios específicos para la vejez de carácter integral, que comprenden desde la prevención de enfermedades y promoción de la salud hasta la rehabilitación y paliación. A ello se une el desarrollo emergente de nuevas formas asistenciales, como la asistencia a domicilio, residencias asistidas o los cuidados paliativos; nuevas especialidades médicas, como la geriatría; o la mayor visibilidad de otras profesiones sanitarias, como los asistentes sociales.

Esto supone la adopción de una estructura de valores capaz de entender y tratar de forma adecuada los aspectos sanitarios asociados al envejecimiento o a la inmigración: tratar pluripatologías, la capacidad de orientación y coordinación de los servicios asistenciales requeridos por los pacientes crónicos, capacitar a los médicos con la competencia cultural apropiada para tratar con respeto los aspectos relacionados con la diversidad étnica, y las diferentes costumbres y valores de los nuevos ciudadanos.

Cambios epidemiológicos: Ha supuesto el cambio del patrón de enfermedades asociado al pasar de un patrón de enfermedad agudo a uno más crónico, a la existencia de comorbilidades, y a la aparición de nuevas enfermedades como consecuencia de la longevidad poblacional y de otros factores, como por ejemplo los movimientos de migración masiva dependientes de los medios de transporte aéreos. La coordinación entre niveles asistenciales aparece como uno de los retos de los profesionales médicos.

Cambios tecnológicos: Incremento del número de innovaciones destinadas a la prevención, diagnóstico y tratamiento de enfermedades; avances en el desarrollo de las tecnologías de la información, la comunicación y sus aplicaciones sanitarias. En la actualidad, los procesos de investigación y desarrollo en tecnologías sanitarias se orientan hacia dos áreas específicas relacionadas de investigación: biotecnología y genética. 
Cambios económicos: La sanidad no es ajena a los procesos de globalización económica y a sus consecuencias. La industrialización de la sanidad ha supuesto la constitución de corporaciones estatales, empresariales y, posiblemente, en el futuro, de usuarios que establecen entre sí negociaciones sobre el modelo de financiación y provisión de servicios sanitarios.

Cambios laborales: La transición laboral de la profesión médica constituye uno de los fenómenos sociales más interesantes en el campo de la sociología de las profesiones.

Cambios mediáticos: Los asuntos éticos atraen la atención pública general y con frecuencia los medios de comunicación cubren el debate sobre ellos. A través de la legislación, medidas administrativas o decisiones jurídicas, los gobiernos cada vez se involucran más en la ética médica.

Cambios políticos y jurídicos: Hay un creciente impacto de la salud en la agenda política. Este impacto aparece asociado a tres factores relacionados entre sí: la importancia de la salud en los medios de comunicación, la sostenibilidad financiera de la sanidad en el contexto del Estado del Bienestar, y el descubrimiento por parte de la clase política de que los temas sanitarios sensibilizan a los ciudadanos y, por lo tanto, pueden ser objeto de valoración electoral prioritaria.

La tendencia a una mayor revisión judicial de la práctica profesional podría ir en aumento en nuestro país imitando lo ocurrido en otros países.

Cambio ético: Los cambios antes descritos han producido, y están produciendo, un cambio importante en el ejercicio de la profesión. En este contexto, la reivindicación del profesionalismo aparece como eje central del contrato social entre medicina y sociedad. El profesionalismo está llamado a reemplazar al modelo más tradicional de contrato social basado en el Juramento Hipocrático.

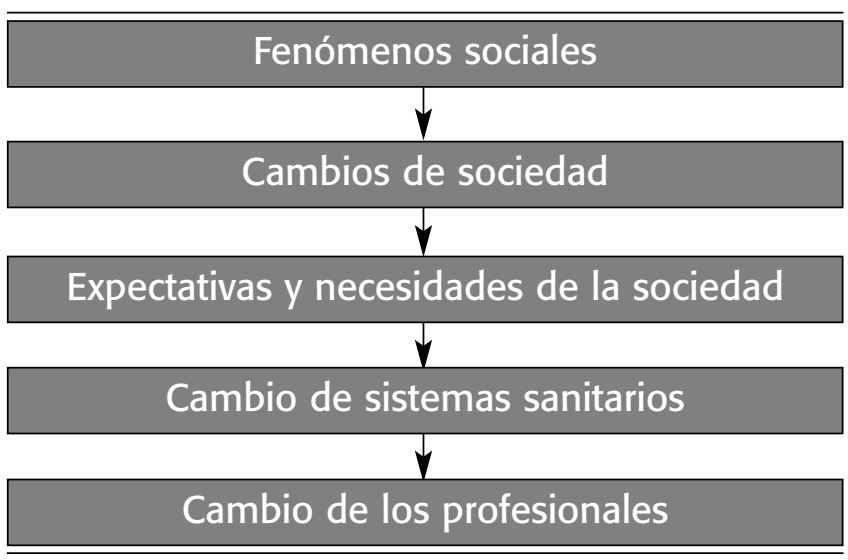

Aunque, a lo largo del mundo, la presión médica se encuentra incrustada en culturas y tradiciones nacionales diversas, sus miembros comparten el papel de sanador, cuyas raíces retroceden hasta Hipócrates. Así, la profesión médica debe contender con complicadas fuerzas políticas, legales y de mercado. Además, existen amplias variaciones en la prestación y la práctica médica a través de las cuales todos los principios generales pueden expresarse en formas a la vez complejas y sutiles.

Ciertos aspectos de la medicina son fundamentales y no cambian con el paso del tiempo, pero el ejercicio de la medicina clínica siempre está cambiando. Los médicos deben estar preparados para hacer frente a los cambios y para reafirmar aquello que es fundamental. Estos cambios exigen que los médicos expresen claramente los principios éticos que guían su conducta, ya sea en la atención clínica, la investigación, la docencia y hasta como ciudadanos. Es crucial que se escuche una perspectiva médica responsable a medida que se toman las decisiones por la sociedad.

La profesionalidad es la base del contrato de la medicina con la sociedad. Exige situar los intereses de los pacientes por encima de los del médico, fijar y mantener unos patrones de competencia y de integridad, y aportar sus conocimientos médicos a la sociedad en materias de salud. Los principios y las responsabilidades de la profesión médica deben ser claramente comprendidos tanto por los médicos como por la sociedad. Para este contrato es esencial la confianza en los profesionales, que se basa en la integridad de los médicos considerados de manera individual y de toda la profesión.

En España, los médicos internos residentes (MIR) antes de entrar en la residencia reciben poca formación en responsabilidad médica, valores y actitudes. La licenciatura de Medicina ofrece un escaso porcentaje de créditos de asignaturas que enseñan a trasmitir estos valores. El sistema de formación de médicos debe, por tanto, adaptarse a una realidad cambiante. Se debería incidir en la transmisión de los principios y los valores, ya que constituyen la piedra angular de la formación de los médicos internos residentes.

Enseñar y transmitir estos valores, forma parte del trabajo de los médicos experimentados. Los médicos tienen la responsabilidad de compartir el conocimiento y la información con sus colegas y pacientes. Esto incluye enseñar las habilidades clínicas y comunicar los resultados de la investigación científica a los compañeros, estudiantes de medicina, médicos residentes y otros proveedores de atención médica. 
El médico tiene la responsabilidad de enseñar la ciencia, el arte y la ética de la medicina a estudiantes de medicina, médicos residentes y otros, así como de supervisar a compañeros médicos en entrenamiento. Los médicos mas experimentados deben tratar a los médicos en entrenamiento con el mismo respeto, empatía y compasión.

Aprender y asimilar es esencial para que un médico en formación llegue a ser un buen profesional. El residente deberá ser capaz de abordar dichos conflictos y tras un proceso de deliberación, ya sea individual o tras la consulta con los recursos destinados a tal fin que estén a su alcance (compañeros, comités de ética asistenciales, comités de los Colegios de Médicos, etc.), valorar las posibles vías de solución e intentar seleccionar las consideradas como óptimas para cada caso.

El residente aprende mientras trabaja y sigue un proceso de progresiva maduración en la que el equilibrio entre formación y responsabilidad de la asistencia es progresivo. Como profesional contrae una serie de responsabilidades con los pacientes, y con la sociedad, que le obligan en su práctica profesional.

Con objeto de mantener la fidelidad con el contrato social de la medicina en estos turbulentos tiempos, los residentes deben reafirmar su dedicación activa a los principios de su profesionalidad. Para ello cuentan con tres principios fundamentales y una serie de responsabilidades profesionales.

\section{PRINCIPIOS FUNDAMENTALES}

Principio de la primacía del bienestar del paciente: Este principio se basa en la entrega al servicio del interés del paciente. El altruismo contribuye a la confianza, que es fundamental en la relación entre paciente y médico.

Principio de la autonomía del paciente: Los médicos deben respetar la autonomía de los pacientes. Los residentes deben ser sinceros con sus pacientes y permitirles que tomen decisiones informadas acerca de su tratamiento. Las decisiones de los pacientes sobre su atención médica deben poseer una importancia primordial, en la medida en que dichas decisiones concuerden con la práctica ética y no puedan resultar en demandas por atención inadecuada.

Principio de la justicia social: La profesión médica debe promover la justicia en el sistema sanitario, lo que incluye una distribución correcta de los recursos sanitarios. Los médicos deben trabajar activamente para eliminar la discriminación en la atención sanitaria, ya se base en la raza, el sexo, el estado socioeconómico, el origen étnico, la religión o cualquier otra categoría social.

\section{RESPONSABILIDADES}

Compromiso de competencia profesional. Los médicos tienen la responsabilidad de seguir acrecentando sus conocimientos a lo largo de toda su vida profesional y de mantener los conocimientos médicos y las actitudes médicas y de equipo necesarias para la prestación de una atención de calidad. De manera más general, la profesión en conjunto debe esforzarse en garantizar que todos sus miembros sean competentes.

Compromiso de honestidad con los pacientes. Los médicos deben asegurar que los pacientes reciban una información completa y honesta antes de que otorguen su consentimiento al tratamiento y una vez que este ha tenido lugar.

Compromiso de la confidencialidad del paciente. Ganar la confianza y el respeto de los pacientes requiere que se apliquen unas salvaguardas de confidencialidad adecuadas a la revelación de la información relacionada con el paciente. Esta responsabilidad se extiende a la discusión con las personas que actúan en representación del paciente cuando no sea posible la obtención del consentimiento por el propio paciente. El cumplimiento de la responsabilidad de confidencialidad es hoy más ineludible que nunca, dada la generalización del uso de sistemas electrónicos de información para la recogida de los datos de los pacientes y la cada vez mayor disponibilidad de información genética.

Compromiso de mantener una relación apropiada con los pacientes. Dadas la vulnerabilidad y dependencia inherentes de los pacientes, deben evitarse ciertas relaciones y prácticas entre médicos y pacientes. En especial, los médicos nunca deben aprovecharse de los pacientes en cuanto a fines de carácter sexual, personal, económico o privado de cualquier otro tipo.

Compromiso con la mejora de la calidad de la atención. Los médicos deben consagrarse a la mejora continua de la calidad de la atención sanitaria. Esta responsabilidad no sólo conlleva mantener la competencia clínica sino también trabajar conjuntamente con otros profesionales para reducir el error médico, incrementar la seguridad de los pacientes, minimizar el uso excesivo de los recursos sanitarios y optimizar el resultado de la atención médica. Los médicos, tanto desde el punto de vista individual como a través de sus asociaciones profe- 
sionales, deben asumir la responsabilidad de ayudar a la creación y la puesta en práctica de mecanismos concebidos para estimular una mejora continua de la calidad de la atención sanitaria.

Compromiso de mejora del acceso a la atención médica. La profesionalidad médica demanda que un objetivo de todos los sistemas de atención sanitaria sea la disponibilidad de un nivel de atención sanitaria uniforme y adecuada. Los médicos, tanto individualmente como colectivamente, deben esforzarse en reducir las barreras que impiden una atención médica equitativa.

Compromiso para una justa distribución de los recursos finitos. A la vez de satisfacer las necesidades de los pacientes individuales, los médicos deben aportar una atención sanitaria basada en un manejo juicioso y rentable de unos recursos clínicos limitados. Deben comprometerse a trabajar con otros médicos, hospitales y sistemas de atención médica para desarrollar unas normas que permitan una atención sanitaria rentable. La responsabilidad del profesional médico en cuanto a la distribución adecuada de los recursos requiere evitar de manera escrupulosa las pruebas y procedimientos superfluos.

Compromiso del conocimiento científico. Gran parte del contrato de la medicina con la sociedad se basa en la integridad y el uso apropiado del conocimiento y de la tecnología científica. Los médicos tienen el deber de mantener su nivel científico, estimular la investigación y crear nuevos conocimientos y asegurar su uso adecuado. La profesión es responsable de la integridad de estos conocimientos, que deben basarse en la evidencia científica y en la experiencia profesional.

Compromiso de mantener la confianza mediante la resolución de los conflictos de intereses. Los profesionales médicos y sus organizaciones se ven enfrentados a numerosas situaciones que pueden comprometer sus responsabilidades profesionales por la persecución de beneficios económicos privados o de ventajas personales. Estas situaciones son especialmente peligrosas en el desarrollo de interacciones personales y organizativas con industrias con fines de lucro, lo que incluye a los fabricantes de equipos sanitarios, las compañías de seguros y las firmas farmacéuticas. Los médicos tienen la obligación de reconocer, revelar al público general y resolver los conflictos de intereses que puedan surgir en el curso de sus funciones y actividades profesionales. Deben revelarse las relaciones entre la industria y los líderes de opinión, en especial, cuando estos últimos determinen los criterios de desarrollo y comunicación de los ensayos clínicos, redacción de editoriales o de normas terapéuticas, o actúen como editores de revistas científicas.

Compromiso de responsabilidades profesionales. Como miembros de una profesión, los médicos deben trabajar conjuntamente para potenciar la atención a los pacientes, ser respetuosos entre sí y participar en los procesos de autorregulación, lo que comprende la corrección y la adopción de medidas disciplinarias en aquellos de sus miembros que no hayan cumplido sus responsabilidades profesionales. La profesión debe definir también y organizar el proceso de formación y de fijación de las normas de conducta para sus miembros actuales y futuros. Los médicos tienen la obligación individual y colectiva de intervenir en estos procesos. Estas obligaciones comprenden la participación en evaluaciones internas y la aceptación del escrutinio y control externos.

La práctica de la medicina en la era moderna se enfrenta a retos sin precedentes en prácticamente todas las culturas y todas las sociedades. Estos retos se deben fundamentalmente al aumento de las disparidades entre las necesidades legítimas de los pacientes, los recursos disponibles para satisfacer dichas necesidades, el aumento de la dependencia en las fuerzas de mercado para transformar el sistema de atención sanitaria, y en la tentación para los médicos de abandonar su compromiso tradicional con la primacía de los intereses de los pacientes. A fin de mantener la fidelidad con el contrato social de la medicina en estos turbulentos tiempos, creemos que los médicos deben reafirmar su dedicación activa a los principios de profesionalidad, lo que supone no solamente el compromiso personal al bienestar de los pacientes sino también el esfuerzo colectivo para mejorar el sistema de atención médica para el bienestar de la sociedad.

\section{BIBLIOGRAFÍA}

1. American Board of Internal Medicine. Project Professionalism. Philadelphia, PA: ABIM, 2000

2. Real Decreto 63/95 de 20 de enero de Ordenación de prestaciones sanitarias del Sistema Nacional de Salud, BOE $n^{\circ} 35$, de 10 de Febrero de 1995

3. Programa de formación de la especialidad de Medicina Interna.

4. El Futuro de la Profesión Médica: análisis del cambio social y los roles de la profesión médica en el siglo XXI Albert J. Jovell Fernández. 